\title{
Hepatosplenic T-Cell Lymphoma Diagnosed by Endoscopic Ultrasound-Guided Fine-Needle Biopsy
}

\author{
Yoshiaki Shibata', Mayuko Miyamoto', Wataru Shinomiya', Kumiko Kirita', Sayuri Motomura², Hiroko Hidai', Takeshi Hagino ${ }^{2}$ \\ and Yuji Ito $^{3}$ \\ Division of ${ }^{1}$ Gastroenterology, ${ }^{2}$ Hematology, ${ }^{3}$ Pathology, Tama-Hokubu Medical Center, Tokyo, Japan
}

Primary splenic malignant lymphoma is very rare, and few reports have examined the diagnostic utility of endoscopic ultrasound-guided fine-needle biopsy (EUS-FNB) for this disease. ${ }^{1-3}$ Here we report an extremely rare case of hepatosplenic T-cell lymphoma (HSTCL). Although HSTCL has been previously documented in patients with inflammatory bowel disease, ${ }^{4,5}$ this is the first report of it being diagnosed by splenic biopsy using EUS-FNB. HSTCL is an extranodal cytotoxic T-cell lymphoma that is located in the liver or spleen, and easily invades the bone marrow; it is characterized by marked hepatosplenomegaly and B symptoms. This disease is more common in young men, and has an aggressive course and poor prognosis. ${ }^{6,7}$

The patient was a 44-year-old woman who was admitted to our hospital with complaints of fever and abdominal pain. Hematologic examination revealed thrombocytopenia, a prolonged blood coagulation time, and an increased serum lactate dehydrogenase level. The platelet count was $73,000 / \mathrm{mm}^{3}$ (normal range, $158,000-348,000 / \mathrm{mm}^{3}$ ), the international normalized ratio of prothrombin time was 1.37 (normal range, 0.85-1.15), the activated partial prothrombin time was $47.1 \mathrm{sec}$ (normal range, $20.0-40.0 \mathrm{sec}$ ), and the serum lactate dehydrogenase level was 593 IU/L (normal

Received: September 18, 2019 Revised: January 19, 2020

Accepted: January 20, 2020

Correspondence: Yoshiaki Shibata

Division of Gastroenterology, Tama-Hokubu Medical Center, Tokyo Metropolitan Health and Medical Treatment Corporation, 1-7-1 Aobacho, Higashimurayamashi, Tokyo 189-0002, Japan

Tel: +81-42-396-3811, Fax: +81-42-396-3076, E-mail: yshibatathk@gmail.com ORCID: https://orcid.org/0000-0002-3559-8546

(c) This is an Open Access article distributed under the terms of the Creative Commons Attribution Non-Commercial License (http://creativecommons.org/ licenses/by-nc/3.0) which permits unrestricted non-commercial use, distribution, and reproduction in any medium, provided the original work is properly cited. range, 124-222 IU/L). Abdominal computed tomography (CT) showed marked splenomegaly (Fig. 1A). ${ }^{18}$ F-Fluorodeoxyglucose (F-FDG) positron emission tomography/CT showed preferential accumulation of ${ }^{18} \mathrm{~F}$-FDG in the spleen (Fig. 1B), as well as in the liver and bone marrow, but there was no accumulation in the lymph nodes. EUS revealed that the spleen was markedly enlarged, with small isoechoic to hypoechoic areas throughout, but no noticeable mass formation was observed (Fig. 1C). The decision was made to perform EUSFNB to confirm a suspected diagnosis of splenic lymphoma. Written informed consent was obtained from the patient after providing a detailed explanation of the procedure. The splenic lesion was punctured transgastrically (Fig. 1C) and the tissue was collected under slight negative pressure using the slow-pull method by slowly withdrawing the stylet. ${ }^{8,9}$ Five punctures were performed using a 22-gauge reverse bevel FNB needle (EchoTip ProCore; Cook Medical, Bloomington, IN, USA) to obtain tissue for immunohistochemistry analysis. There were no procedure-related adverse events. Histopathologic analysis showed diffuse infiltration of medium-sized atypical lymphocytes, with clear cytoplasm, into the sinusoids of the spleen (Fig. 2A). Immunohistochemistry analysis revealed markedly abnormal lymphoid infiltrates in the splenic sinuses that were positive for $\mathrm{CD} 3$ (Fig. 2B) and negative for CD4 (Fig. 2C), CD8 (Fig. 2D), and CD20 (Fig. 2E), which is characteristic of HSTCL. The results of bone marrow analysis using flow cytometry also supported this diagnosis, and this case was found to be of the $\alpha \beta$ T-cell receptor type (with the $\alpha \beta$ type being less dominant than the $\gamma \delta$ type). ${ }^{6,7}$ Using a 22-gauge core biopsy needle allowed the collection of a tissue sample with a length of $579.4 \mu \mathrm{m}$, which was sufficient to meet the fifth criterion (sufficient material for good quality histological interpretation) in the standard microscopic scor- 
ing system proposed by Gerke et al. ${ }^{10}$ A 19-gauge core biopsy needle is useful for diagnosis of splenic malignant lymphoma by EUS-FNB because a large amount of tissue can be collected with a small number of punctures, ${ }^{2,3}$ but procedures involving an EUS scope sometimes require a strong upward angle and rightward torque to detect and puncture splenic lesions. A 22-gauge biopsy needle is more flexible than a 19-gauge needle, therefore less resistance is encountered when attaching the needle and puncturing the target in such situations. This makes it easier for less experienced operators to control the needle and puncture the target. Therefore, we opted to use a 22-gauge core biopsy needle for splenic biopsy in this case.

The spleen is the primary site of non-Hodgkin's lymphoma in only $1 \%-3 \%$ of the cases. ${ }^{6,7}$ Furthermore, HSTCL accounts for less than $1 \%$ of all non-Hodgkin's lymphomas. ${ }^{6,7}$ Interestingly, $10 \%$ of the known cases of HSTCL have been documented in patients with inflammatory bowel disease, such as Crohn's disease or ulcerative colitis. ${ }^{4,5}$ There have also been some reports of cases where immunosuppressants, such as anti-tumor necrosis factor (TNF) and thiopurine, have been used in combination. ${ }^{4,5}$ Concomitant use of thiopurine and anti-TNF is thought to cause bone marrow suppression, resulting in an inability to selectively control cell proliferation and development of HSTCL via a genetic mechanism. ${ }^{4}$ Our patient did not have a history of inflammatory bowel disease or immunosuppressive therapy. Immunosuppressants such as anti-TNF and thiopurine are now widely used in inflammatory bowel disease, and patients receiving these agents should be monitored carefully for development of HSTCL. Splenic biopsy with EUS using a core biopsy needle, which is a minimally invasive procedure, may aid in early diagnosis of HSTCL in patients with inflammatory bowel disease receiving immuno-
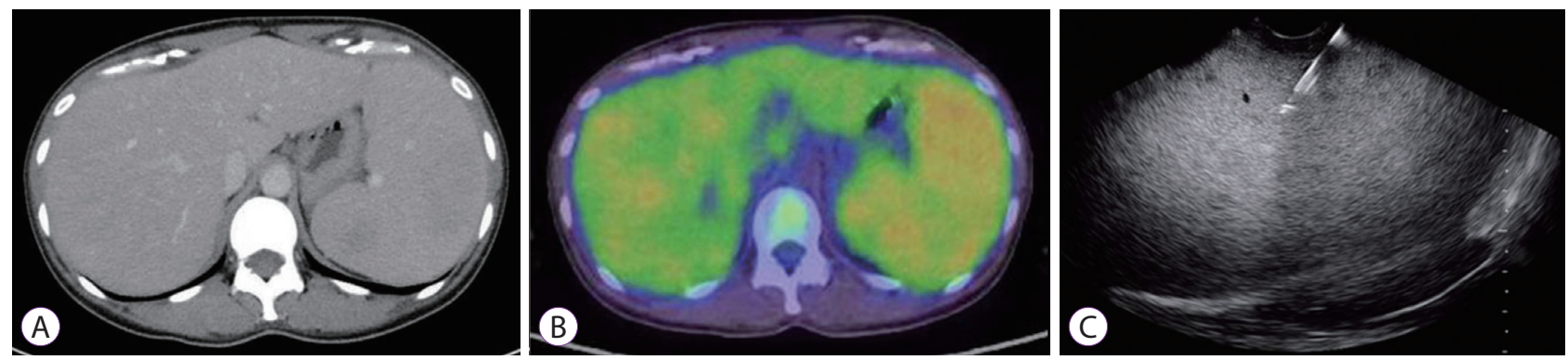

Fig. 1. Abdominal computed tomography (CT), ${ }^{18} \mathrm{~F}$-Fluorodeoxyglucose (F-FDG) positron emission tomography (PET)/CT, and endoscopic ultrasound images for a patient with hepatosplenic T-cell lymphoma. (A) Abdominal CT scan showing marked splenomegaly. (B) ${ }^{18} \mathrm{~F}$-FDG PET/CT scan showing abnormal accumulation of FDG in the spleen. (C) Endoscopic ultrasound images showing marked splenomegaly but no noticeable mass formation. The spleen was punctured by a 22 -gauge biopsy needle.
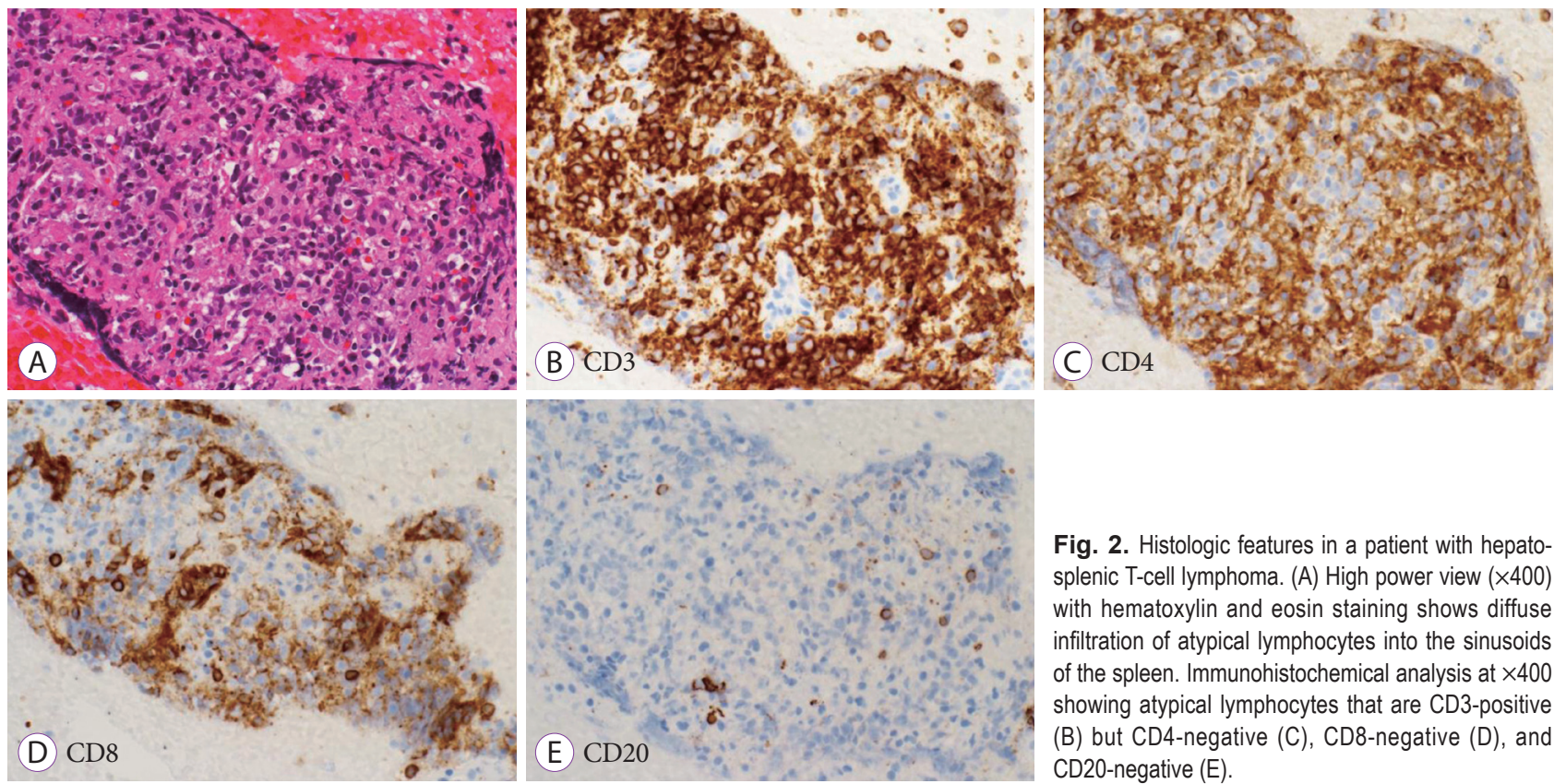

Fig. 2. Histologic features in a patient with hepatosplenic T-cell lymphoma. (A) High power view $(\times 400)$ with hematoxylin and eosin staining shows diffuse infiltration of atypical lymphocytes into the sinusoids of the spleen. Immunohistochemical analysis at $\times 400$ showing atypical lymphocytes that are CD3-positive (B) but CD4-negative (C), CD8-negative (D), and CD20-negative (E). 
suppressive therapy.

Conflicts of Interest

The authors have no financial conflicts of interest.

\section{Acknowledgment}

The authors thank Dr. Shigeo Mori, Professor Emeritus at The University of Tokyo, for advice on the pathology of HSTCL.

\author{
Author Contributions \\ Conceptualization: Yoshiaki Shibata, Sayuri Motomura, Hiroko Hidai, \\ Takeshi Hagino \\ Data curation: Mayuko Miyamoto, SM, HH, TH \\ Formal analysis: YS, Yuji Ito \\ Investigation: MM, Wataru Shinomiya, Kumiko Kirita \\ Methodology: YS, YI \\ Project administration: YS, YI \\ Supervision: YS, SM, YI \\ Validation: MM, WS, KK, SM, HH, TH, YI \\ Writing-original draft: YS \\ Writing-review\&editing: YS, MM, WS, KK, SM, HH, TH, YI
}

\section{ORCID}

Mayuko Miyamoto: https://orcid.org/0000-0001-8828-4658

Wataru Shinomiya: https://orcid.org/0000-0001-6886-3345

Kumiko Kirita: https://orcid.org/0000-0003-2960-6690

Sayuri Motomura: https://orcid.org/0000-0002-6960-0800

Hiroko Hidai: https://orcid.org/0000-0003-2684-3586

Takeshi Hagino: https://orcid.org/0000-0002-3164-9641

Yuji Ito: https://orcid.org/0000-0002-5776-1447

\section{REFERENCES}

1. Eloubeidi MA, Varadarajulu S, Eltoum I, Jhala D, Chhieng DC, Jhala NC. Transgastric endoscopic ultrasound-guided fine-needle aspiration biopsy and flow cytometry of suspected lymphoma of the spleen. Endoscopy 2006;38:617-620.

2. Iwashita T, Yasuda I, Tsurumi H, et al. Endoscopic ultrasound-guided fine needle aspiration biopsy for splenic tumor: a case series. Endoscopy 2009;41:179-182.

3. Saab S, Challita Y, Holloman D, Hathaway K, Kahaleh M, Nieto J. Case series review of the safety and efficacy of endoscopic ultrasound-guided splenic mass core biopsy. Clin Endosc 2018;51:600-601.

4. Thai A, Prindiville T. Hepatosplenic T-cell lymphoma and inflammatory bowel disease. J Crohns Colitis 2010;4:511-522.

5. Deepak P, Sifuentes H, Sherid M, Stobaugh D, Sadozai Y, Ehrenpreis ED. T-cell non-Hodgkin's lymphomas reported to the FDA AERS with tumor necrosis factor-alpha (TNF-alpha) inhibitors: results of the REFURBISH study. Am J Gastroenterol 2013;108:99-105.

6. Macon WR, Levy NB, Kurtin PJ, et al. Hepatosplenic alphabeta T-cell lymphomas: a report of 14 cases and comparison with hepatosplenic gammadelta T-cell lymphomas. Am J Surg Pathol 2001;25:285-296.

7. Yabe M, Miranda RN, Medeiros LJ. Hepatosplenic T-cell lymphoma: a review of clinicopathologic features, pathogenesis, and prognostic factors. Hum Pathol 2018;74:5-16.

8. Kudo T, Kawakami H, Hayashi T, et al. High and low negative pressure suction techniques in EUS-guided fine-needle tissue acquisition by using 25-gauge needles: a multicenter, prospective, randomized, controlled trial. Gastrointest Endosc 2014;80:1030-1037.e1.

9. Nakai $\mathrm{Y}$, Isayama $\mathrm{H}$, Chang $\mathrm{KJ}$, et al. Slow pull versus suction in endoscopic ultrasound-guided fine-needle aspiration of pancreatic solid masses. Dig Dis Sci 2014;59:1578-1585.

10. Gerke H, Rizk MK, Vanderheyden AD, Jensen CS. Randomized study comparing endoscopic ultrasound-guided Trucut biopsy and fine needle aspiration with high suction. Cytopathology 2010;21:44-51. 